Review Article

\title{
Chinese Herbal Bath Therapy for the Treatment of Knee Osteoarthritis: Meta-Analysis of Randomized Controlled Trials
}

\author{
Bo Chen, ${ }^{1}$ Hongsheng Zhan, ${ }^{1}$ Mei Chung, ${ }^{2}$ Xun Lin, ${ }^{1}$ Min Zhang, \\ Jian Pang, ${ }^{1}$ and Chenchen Wang ${ }^{3}$ \\ ${ }^{1}$ Research Institute of Orthopedics \& Traumatology, Shuguang Hospital affiliated to Shanghai University of \\ Traditional Chinese Medicine, Shanghai, China \\ ${ }^{2}$ Department of Public Health and Community Medicine, School of Medicine, Tufts University, Boston, MA, USA \\ ${ }^{3}$ Center for Complementary and Integrative Medicine, Division of Rheumatology, Tufts Medical Center, \\ Tufts University School of Medicine, Boston, MA, USA
}

Correspondence should be addressed to Chenchen Wang; cwang2@tuftsmedicalcenter.org

Received 20 April 2015; Accepted 8 June 2015

Academic Editor: Andreas Sandner-Kiesling

Copyright (C) 2015 Bo Chen et al. This is an open access article distributed under the Creative Commons Attribution License, which permits unrestricted use, distribution, and reproduction in any medium, provided the original work is properly cited.

Objective. Chinese herbal bath therapy (CHBT) has traditionally been considered to have analgesic and anti-inflammatory effects. We conducted the first meta-analysis evaluating its benefits for patients with knee osteoarthritis (OA). Methods. We searched three English and four Chinese databases through October, 2014. Randomized trials evaluating at least 2 weeks of CHBT for knee OA were selected. The effects of CHBT on clinical symptoms included both pain level (via the visual analog scale) and total effectiveness rate, which assessed pain, physical performance, and wellness. We performed random-effects meta-analyses using mean difference. Results. Fifteen studies totaling 1618 subjects met eligibility criteria. Bath prescription included, on average, 13 Chinese herbs with directions to steam and wash around the knee for 20-40 minutes once or twice daily. Mean treatment duration was 3 weeks. Results from meta-analysis showed superior pain improvement (mean difference $=-0.59$ points; $95 \%$ confidence intervals [CI], -0.83 to $-0.36 ; p<0.00001$ ) and higher total effectiveness rate (risk ratio $=1.21 ; 95 \%$ CI, 1.15 to $1.28 ; p<0.00001$ ) when compared with standard western treatment. No serious adverse events were reported. Conclusion. Chinese herbal bath therapy may be a safe, effective, and simple alternative treatment modality for knee OA. Further rigorously designed, randomized trials are warranted.

\section{Introduction}

Knee osteoarthritis $(\mathrm{OA})$ is one of the most common diseases of chronic joint pain among aging populations [1]. It is associated with physical and psychosocial disability, reduced quality of life, and substantial health care costs $[2,3]$. Currently, no effective disease-modifying remedies are available to treat knee OA [4].Complementary and alternative medicine therapies have been heavily advertised, and there are an increasing number of patients with chronic pain who report utilizing these treatments [5].

As an ancient traditional treatment, herbal bath therapy has developed over thousands of years in China. In the earliest published Chinese medical work "Inner Classic of the Yellow Emperor" (475 B.C.-221 B.C.) and "Prescriptions for fifty-two diseases" (202 B.C.-9 A.D.), herbal steaming and washing therapy has frequently reported beneficial outcomes. Importantly, herbal bath therapy continues to increase in popularity in Asian countries today, especially for treating chronic muscle and skeletal disorders including knee OA.

Compared to balneotherapy, a spa treatment undertaken in heated mineral water to alleviate musculoskeletal problems in European and Middle Eastern citizens [6,7], herbal bath therapies consist of specific medicinal ingredients targeted to specific symptom differentiation. Chinese herbal bath therapy is believed to have analgesic and anti-inflammatory effects that actively ameliorate symptoms of pain [8] and activate blood circulation [9]. 
Although Chinese herbal bath therapy has been employed to treat arthritis for thousands of years in China, the necessary quantitative evidence to estimate treatment effects is still lacking. No meta-analysis addressing any treatment outcomes of Chinese herbal bath therapy has ever been published [10]. To better inform patients and physicians, we systematically reviewed the medical literature and performed meta-analysis on randomized controlled trials of Chinese herbal bath therapy focusing on knee OA treatment.

To our knowledge, this meta-analysis is the first attempt to systematically analyze all previously published controlled trials of Chinese herbal bath treatment for knee OA patients. Based on these findings, recommendations for clinical practice are offered.

\section{Materials and Methods}

2.1. Search Strategy. A comprehensive search strategy was designed to capture all available literature. We searched PubMed, the Cochrane Library, the Springer Database, the Chinese National Knowledge Infrastructure Database, the Chongqing VIP Database, the Chinese Biomedical Database, and the Wanfang Database up to October 1, 2014. The search terms included "Drugs, Chinese Herbal," "Medicine, Chinese," "Steam Bath," and "baths" as Medical Subject Heading Terms and "knee osteoarthritis" as a keyword. These search terms were adapted and used to search the Cochrane Library and the Springer Database. In Chinese databases, we employed "bath," "steam," "washing," "external use," and "knee Osteoarthritis" as the major search terms with no limitations. In addition, we searched records from the Shanghai University of Traditional Chinese Medicine library.

2.2. Eligibility Criteria. Trials were eligible if they were randomized controlled trials (RCTs) recruiting participants with knee OA, whose intervention included Chinese herbal bath therapy for the duration of at least 2 weeks with more than 10 subjects in each group and if they represented original data. There was no language restriction in the literature search.

In order to evaluate the independent effects of the Chinese herbal bath intervention, we excluded (1) review articles, (2) treatment groups that included nonsteroidal antiinflammatory drugs (NSAIDs), glucosamine, intra-articular injection or surgery, and (3) any control group that included traditional Chinese therapies.

2.3. Selection of Studies. Two authors (BC and XL) independently screened all potential eligible studies. Titles and abstracts were first screened to exclude irrelevant citations. Full text of all articles of potentially relevant abstracts were retrieved and screened according to the study eligibility criteria. The diagnostic criterion was from the American College of Rheumatology 1986 [28]. We also accepted the criteria of the Chinese Orthopedic Association 2007 [26] and Traditional Chinese Medicine 1994 [27] (Table 1 footnotes).

The effects of herbal bath therapy on clinical symptoms were measured by pain level (via the visual analog scale) and total effectiveness rate that assessed pain, physical performance, and wellness. The test-retest reliability and validity of these measures have been demonstrated in patients with arthritis $[29,30]$. The definition and measurement of the outcome measures are described in Table 1.

2.4. Data Extraction. One author (BC) extracted the data from included studies using a predesigned data extraction table. The accuracy of the data extraction was verified by another author (MZ). Study characteristics that were extracted included publication information, origin of study, study setting, time frame of study, age, gender, definition of knee OA, detailed information of interventions and controls, outcome measures, summary of results, main conclusion, and adverse reactions (Table 1 ).

2.5. Statistical Analysis. Included studies were synthesized based on two categories of treatment outcomes: pain score and total effectiveness rate. For meta-analysis of pain score, we combined studies using mean difference (MD) in the VAS score. VAS score ranged from 0 points (no pain) to 10 points (worst possible pain). MD was calculated by subtracting after from before measurements and standard deviation (SD) for change was estimated by the given SD of before and after treatment. A positive mean difference in VAS score indicates an effect favorable to Chinese herbal bath therapy compared with controls.

For meta-analysis of total effectiveness rate, we combined studies using risk ratio comparing Chinese herbal bath therapy with controls. A risk ratio of total effectiveness rate greater than 1 indicates that Chinese herbal bath therapy is more effective than controls, consistent with the direction of VAS score.

In view of significant clinical heterogeneity, the DerSimonian-Laird random-effects model was used for pooling [31]. Statistical heterogeneity across included studies was estimated using the Cochran Q statistic (considered significant when the $p$ value was less than 0.10 ) and quantified the extent of heterogeneity with the $I^{2}$ index [32]. All analyses were conducted using RevMan V5.3 (The Nordic Cochrane Centre, The Cochrane Collaboration). All reported $p$ values were two sided and a $p$ value $<0.05$ was considered to be statistically significant.

2.6. Quality Assessment. We independently evaluated the methodological quality of all included studies (BC and JP). Any disagreement between the investigators was resolved with mutual consensus in the presence of the third author (CW). Risk of bias was based on the modified set of criteria adapted from the Newcastle-Ottawa Scale [33] which covered the following items: adequacy of randomization; allocation concealment; similarity of study groups at baseline; blinding; equal treatment of groups throughout the study; completeness of follow-up; and intention to treat (participants analyzed in the groups to which they were randomly assigned) [34]. 


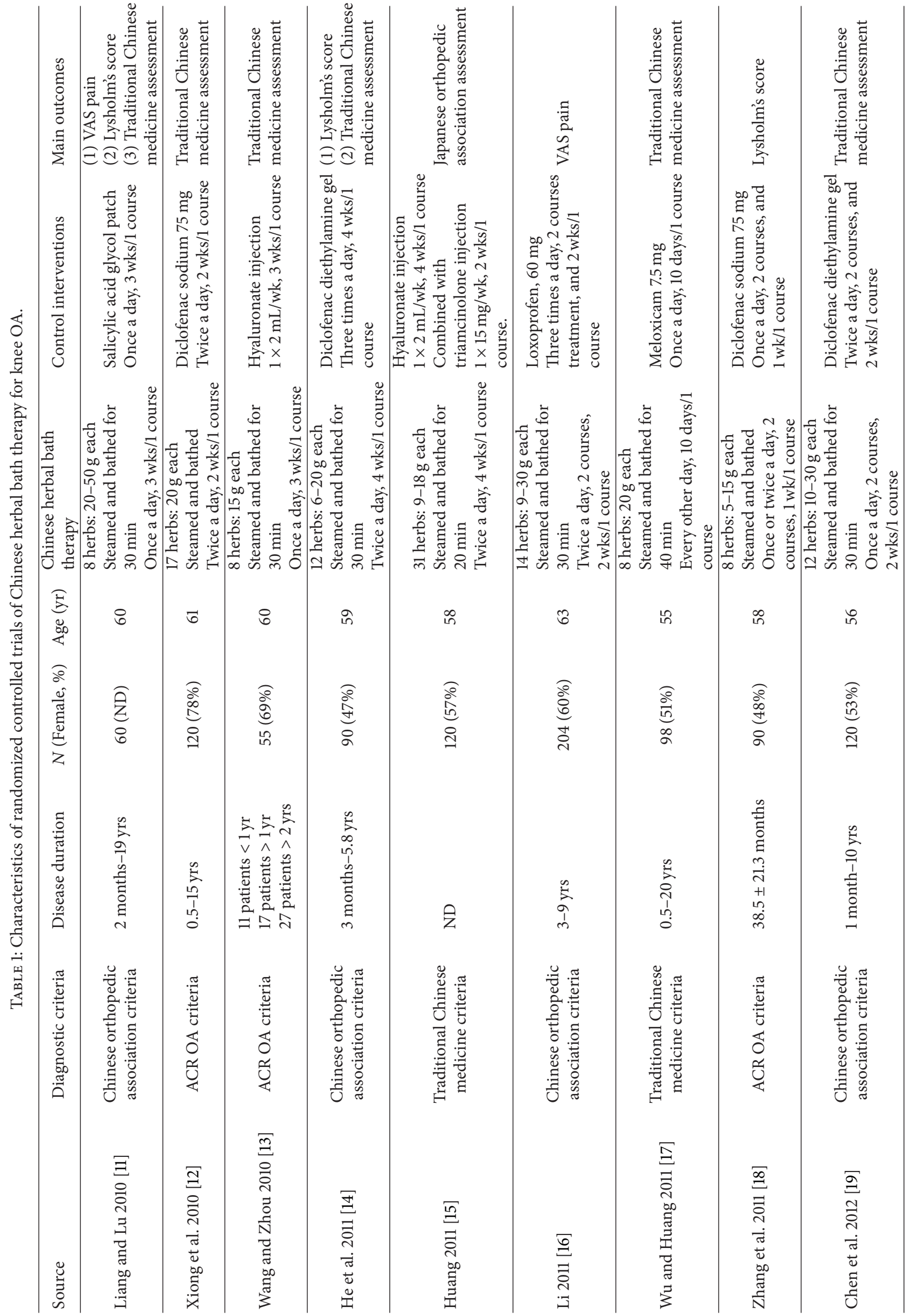




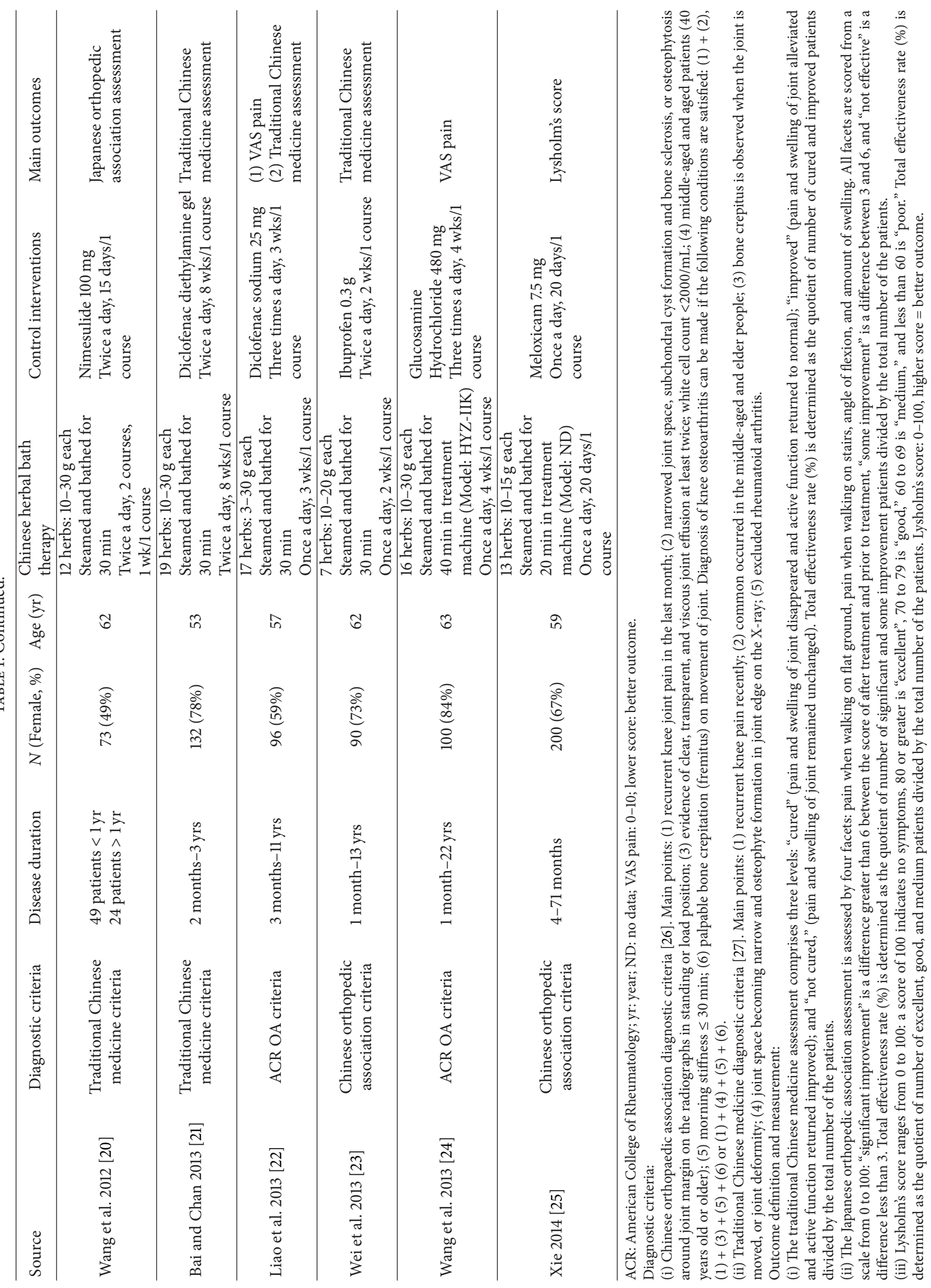




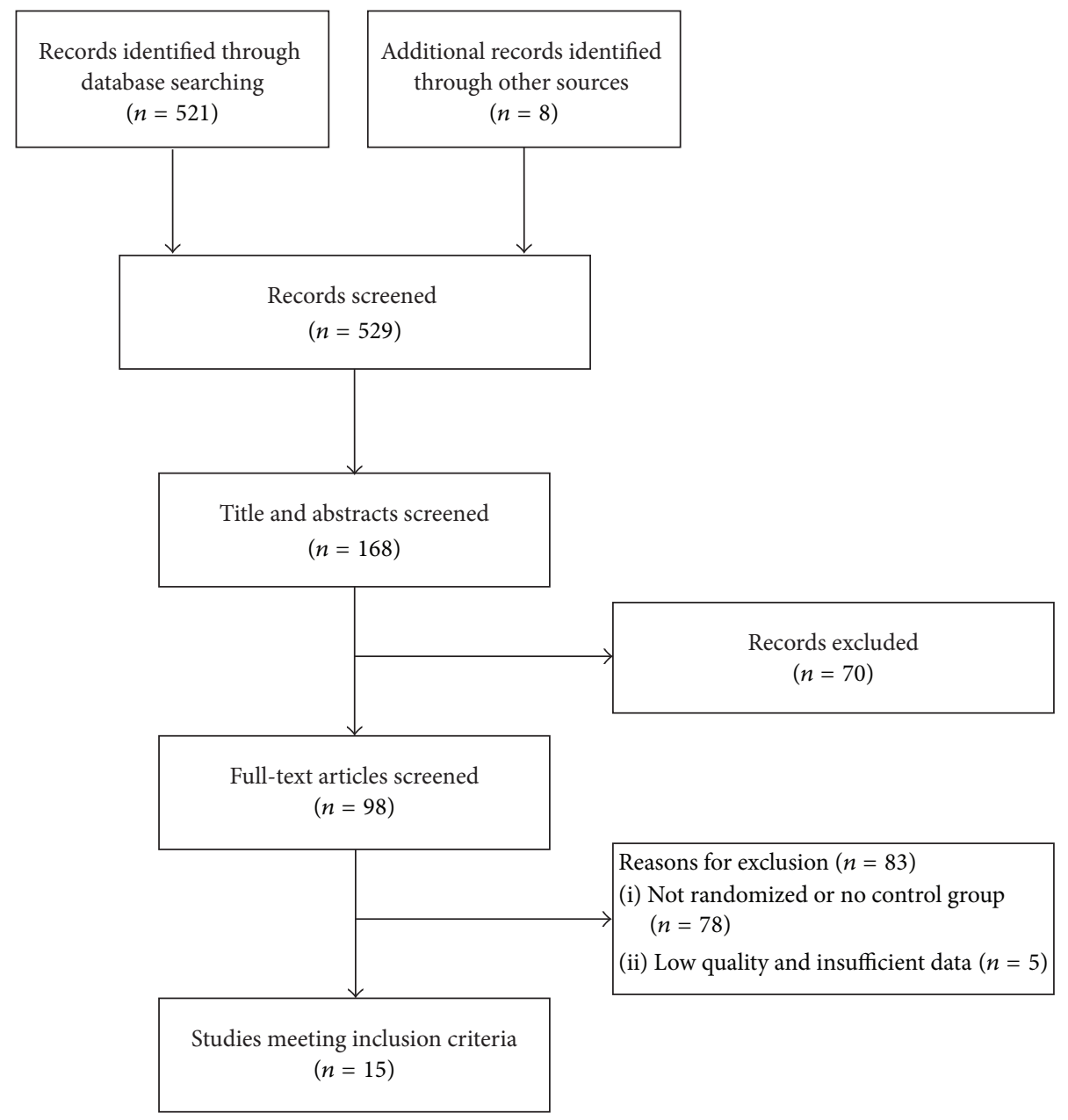

FIGURE 1: Study selection flow chart.

\section{Results}

We screened a total of 529 abstracts identified from 7 English and Chinese databases $(n=521)$. We also searched additional records from Shanghai University of Traditional Chinese Medicine library $(n=8)$. After initially screening 168 potentially relevant abstracts, we excluded 70 because they did not meet the inclusion criteria (i.e., participants did not have knee OA, reviews, case reports, or duplicate publications). We retrieved and reviewed 98 full articles; 83 were excluded due to lack of randomization or absence of a control group ( $n=78)$, major methodologic flaws, and/or insufficient data $(n=5)$. Finally, 15 eligible RCTs [11-25] involving 1618 patients were included. Figure 1 summarizes the detailed study selection process.

3.1. Included Studies. The characteristics of the $15 \mathrm{RCTs}$ are summarized in Table 2. All 15 RCTs were conducted in China and were published between 2010 and 2014. There are a total of 1618 patients (63\% female) with knee OA. Mean age of participants was 59 years and mean symptom duration was 68 months.
On average, a bath prescription in the intervention groups included 13 Chinese herbs, ranging from 7 to 31 . The top 20 frequently prescribed Chinese herbs and efficacy in the total of 15 bath prescriptions are summarized in Table 2. Nine Chinese herbs (Garden Balsam Stem, Common Clubmoss Herb, Clematis Root, Bark of Himalayan Coralbean, Doubleteeth Pubescent Angilica Root, Common Floweringquince Fruit, Slenderstyle Acanthopanax Bark, Divaricate Saposhnikovia Root, and Manchurian Wildginger) claimed an efficacy of pain relief. Six Chinese herbs (Safflower, Twotooth Achyranthes Root, Chinese Angelica, Suberect Spatholobus Stem, Sappan Wood, and Szechuan Lovage Rhizome) claimed an efficacy of activating blood circulation. Five Chinese herbs (Common Monkshood Mother Root, Cassia Twig, Kusnezoff Monkshood Root, Pricklyash Peel, and Argy Wormwood Leaf ) claimed an efficacy of antirheumatic agents. Six Chinese herbs such as Common Clubmoss Herb, Doubleteeth Pubescent Angilica Root, Clematis Root, Safflower, Chinese Angelica, and Argy Wormwood Leaf claimed anti-inflammatory effects $[35,36]$. The duration of treatment ranged from 20 to 40 minutes, once or twice a day. The control groups used NSAIDs, glucosamine, and intra-articular hyaluronate injection for 
TABLE 2: Top 20 Chinese herbs and efficacy according to the frequency of usage in 15 bath prescriptions.

\begin{tabular}{|c|c|c|c|}
\hline English name & Latin name & Chinese Pinyin name & Frequency of usage \\
\hline \multicolumn{4}{|l|}{ Pain relief } \\
\hline Garden Balsam Stem & Caulis Impatientis & Tougucao & 11 \\
\hline Common Clubmoss Herb* & Herba Lycopodii & Shenjincao & 9 \\
\hline Clematis Root ${ }^{*}$ & Radix Clematidis & Weilingxian & 8 \\
\hline Bark of Himalayan Coralbean & Erythrina variegata & Haitongpi & 6 \\
\hline Doubleteeth Pubescent Angilica Root* & Radix Angelicae Pubescentis & Duhuo & 6 \\
\hline Common Floweringquince Fruit & Fructus Chaenomelis & Mugua & 6 \\
\hline Slenderstyle Acanthopanax Bark & Cortex Acanthopanacis & Wujiapi & 5 \\
\hline Divaricate Saposhnikovia Root & Radix Saposhnikoviae & Fangfeng & 5 \\
\hline Manchurian Wildginger & Herba Asari & Xixin & 4 \\
\hline \multicolumn{4}{|l|}{ Activating blood circulation } \\
\hline Safflower* & Flos Carthami & Honghua & 10 \\
\hline Twotooth Achyranthes Root & Radix Achyranthis Bidentatae & Niuxi & 8 \\
\hline Chinese Angelica* & Radix Angelicae Sinensis & Danggui & 8 \\
\hline Suberect Spatholobus Stem & Caulis Spatholobi & Jixueteng & 5 \\
\hline Sappan Wood & Lignum Sappan & Sumu & 4 \\
\hline Szechuan Lovage Rhizome & Rhizoma Chuanxiong & Chuanxiong & 4 \\
\hline \multicolumn{4}{|l|}{ Anti-rheumatic effects } \\
\hline Common Monkshood Mother Root & Radix Aconiti & Chuanwu & 8 \\
\hline Cassia Twig & Ramulus Cinnamomi & Guizhi & 8 \\
\hline Kusnezoff Monkshood Root & Radix Aconiti Kusnezoffii & Caowu & 7 \\
\hline Pricklyash Peel & Fructus Zanthoxyli & Huajiao & 7 \\
\hline Argy Wormwood Leaf* & Folium Artemisiae Argyi & Aiye & 6 \\
\hline
\end{tabular}

${ }^{*}$ These herbs are thought to have anti-inflammatory effects.

treatment. NSAIDs included diclofenac, loxoprofen, meloxicam, nimesulide, ibuprofen, or salicylic acid glycol patch, once to three times a day. Glucosamine was prescribed three times a day while intra-articular hyaluronate injection was given once a week. Mean treatment duration was 3.3 weeks (range 2-8 weeks) for 1-2 courses of treatment in both groups.

3.2. Meta-Analysis. In the fifteen eligible RCTs, four trials $[11,16,22,24]$ measured pain using a VAS scale $(0-10$ points), while thirteen trials [11-15, 17-23, 25] evaluated clinical efficacy via total effectiveness rate. Two trials $[11,22]$ measured and evaluated pain and total effectiveness rates simultaneously.

(1) Pain Outcomes. Four trials [11, 16, 22, 24] involving 460 patients measured pain score based on a VAS scale (0-10 points). The random effects model was used for statistical analysis. The pooled analysis indicated that patients in the Chinese herbal bath therapy groups had significantly lower pain scores than those in the NSAIDs, glucosamine, and intra-articular hyaluronate injection control groups $(\mathrm{MD}=$ $-0.59 ; 95 \%$ confidence intervals [CI], -0.83 to $-0.36 ; p<$ 0.00001 ) after $2-8$ weeks of treatment. There was no evidence for statistical heterogeneity across studies (chi-square $=1.56$; degree of freedom $=3 ; I^{2}=0 \%$ ) (Figure 2). On average, patients in the Chinese herbal bath therapy had significantly lower pain scores than those in the control groups.
(2) Total Effectiveness Rate Outcomes. Thirteen trials [11$15,17-23,25]$ involving 1314 patients reported data on the total effectiveness rate of Chinese herbal bath therapy compared with NSAIDs, glucosamine, and intra-articular hyaluronate injection controls. Nine trials [11-14, 17, 19, 21-23] evaluated the measured outcome on the basis of the Traditional Chinese Medicine criteria; two trials $[15,20]$ evaluated the measured outcome on the basis of the Japanese Orthopedic Association criteria; other two trials $[18,25]$ evaluated the measured outcome on the basis of the Lysholm's score. The results from our random-effects model meta-analysis indicate that Chinese herbal bath therapy on average improved the clinical effective rates by $21 \%$ when compared with controls (risk ratio $[\mathrm{RR}]=1.21 ; 95 \% \mathrm{CI}, 1.15$ to $1.28 ; p<0.00001)$. There was a small degree of statistical heterogeneity across studies $\left(I^{2}=\right.$ $21 \%)$. Our meta-analysis showed that 2-8 weeks of Chinese herbal bath therapy does improve the clinical symptom such as pain, physical performance, and wellness for patients with knee OA.

Further subgroup analysis exploring the improvement of different controls on total effectiveness rate showed that Chinese herbal bath therapy has a better effect compared with NSAIDs ( $\mathrm{RR}=1.21 ; 95 \% \mathrm{CI}, 1.14$ to $1.28 ; p<0.00001)$ and intra-articular hyaluronate injection $(\mathrm{RR}=1.26$; $95 \% \mathrm{CI}$, 1.11 to $1.42 ; p=0.0003$ ) (Figure $3(\mathrm{a})$ ). We also performed a subgroup meta-analysis on total effectiveness rate by the three different assessment outcomes. The results showed similar 


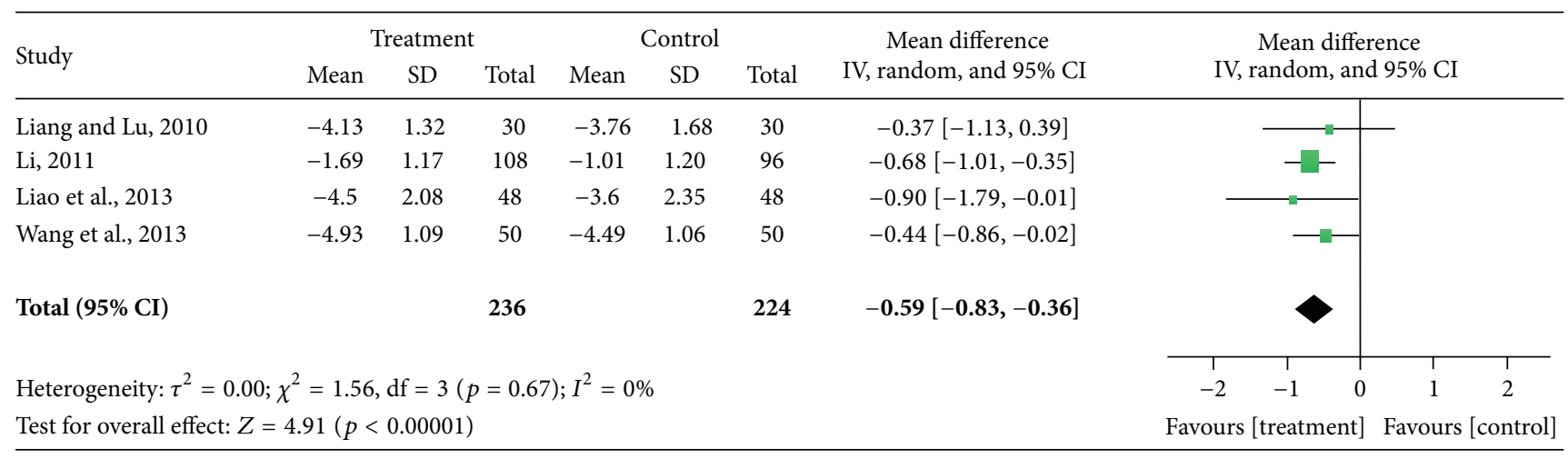

Figure 2: Effect of Chinese herbal bath therapy on pain score (VAS).

results among subgroups (Traditional Chinese Medicine criteria $(\mathrm{RR}=1.24 ; 95 \% \mathrm{CI}, 1.16$ to $1.33 ; p<0.00001)$, JOA criteria $(\mathrm{RR}=1.18 ; 95 \% \mathrm{CI}, 1.07$ to $1.31 ; p<0.01)$, and Lysholm's score $[\mathrm{RR}=1.17$; 95\% CI, 1.02 to $1.33 ; p<0.05]$ ) (Figure 3(b)).

Overall, compared with nonsteroidal anti-inflammatory drugs, glucosamine, and intra-articular hyaluronate injection, all studies reported a positive association range from 2 to 8 weeks of herbal medicated bath therapy and improved clinical symptoms with lower risk of adverse events compared with western medication.

3.3. Adverse Events. Seven trials mentioned adverse events but no serious adverse events were reported. Li reported 18 patients had gastrointestinal symptoms in loxoprofen group but none in the herbal bath group [16]. Wei et al. stated that five patients had gastrointestinal symptoms in the ibuprofen group but none in the herbal bath group [23]. Importantly, Xie reported one patient had cutaneous pruritus in the herbal bath group while there were eight reported in the meloxicam group which included nausea, poor appetite, stomach ache, and skin irritation [25].

3.4. Quality Assessment. The quality assessment of the trials was performed using the Newcastle-Ottawa Scale. The detailed results are presented in Figure 4 . The overall quality of trials was moderate. Randomization was adequate in 4 trials $(26.7 \%)$ and unclear in 11 trials (73\%). All studies reported the similarity of study groups at baseline (100\%). Outcome assessors blinded in 1 trials (6.7\%), unclear in 14 trials $(93.3 \%)$. The bias of blinding to patients, allocation concealment, and intention to treat items were similarly difficult to assess from reported information.

\section{Discussion}

This first systemic review and meta-analysis of 15 RCTs in 1618 individuals indicate that herbal bath therapy has greater beneficial effects than standard western medication for knee OA. Overall, Chinese herbal bath therapy appears to be safe and effective for people who suffer with knee OA.
These findings agree with six recent reviews of balneotherapy. For example, Falagas et al. reported that 29 trials using balneotherapy as therapy for two weeks to one year may be associated with improvement in several rheumatological diseases compared with NSAIDs and other analgesics [37]. Another review of 9 RCTs by Harzy et al. suggested that shortand long-term therapeutic thermal mineral water appears to show some advantage for treating knee OA compared to NSAIDs and analgesics [38]. Additional 4 reviews have shown the effectiveness of balneotherapy including spa therapy at the Dead Sea and Tiberias in Israel for patients with OA [39-42]. Furthermore, mud-bath therapy with Sillene mineral water improved patients with knee OA and significantly reduced the frequency and severity of symptoms and disability [43]. Recently, the importance of the balneotherapy has also been synthesized by the Osteoarthritis Research Society International guidelines [10]. However, balneotherapy treatments are limited to the general use of spa treatment and mineral baths, since no specific medicinal ingredients have been identified that could actively alleviate symptoms of pain and activate blood circulation. In addition, unique treatments such as Dead Sea bath therapy are extremely difficult to generalize and replicate in large populations.

Despite the lack of knowledge about the biologic mechanisms by which Chinese herbal bath therapy work for knee $\mathrm{OA}$, the synergy between the efficacy of herbs and heating power likely plays a major role in symptom management. First, the Chinese medicated herbs, which contain ingredients that promote pain relief, promote flow of Qi (vital energy), reduce swelling and remove blood stasis, bring more nutrients and oxygen to the healing tissues, and energize the antirheumatic effects for the knee joints [44]. Second, the Chinese medicated herbs may also directly act on the injured and degenerative articular cartilage through percutaneous absorption based on a recent report [45]. Third, the heating power of the water temperature itself has the potentiality to improve clinical symptoms [38]. Fourth, recent studies have suggested that local inflammation plays a prominent role in OA's pathogenesis [46, 47]. Several studies have already shown an association of Chinese medicated herbs and the expression of anti-inflammatory cytokines [48-53]. For example, Common Clubmoss Herb and Doubleteeth Pubescent 


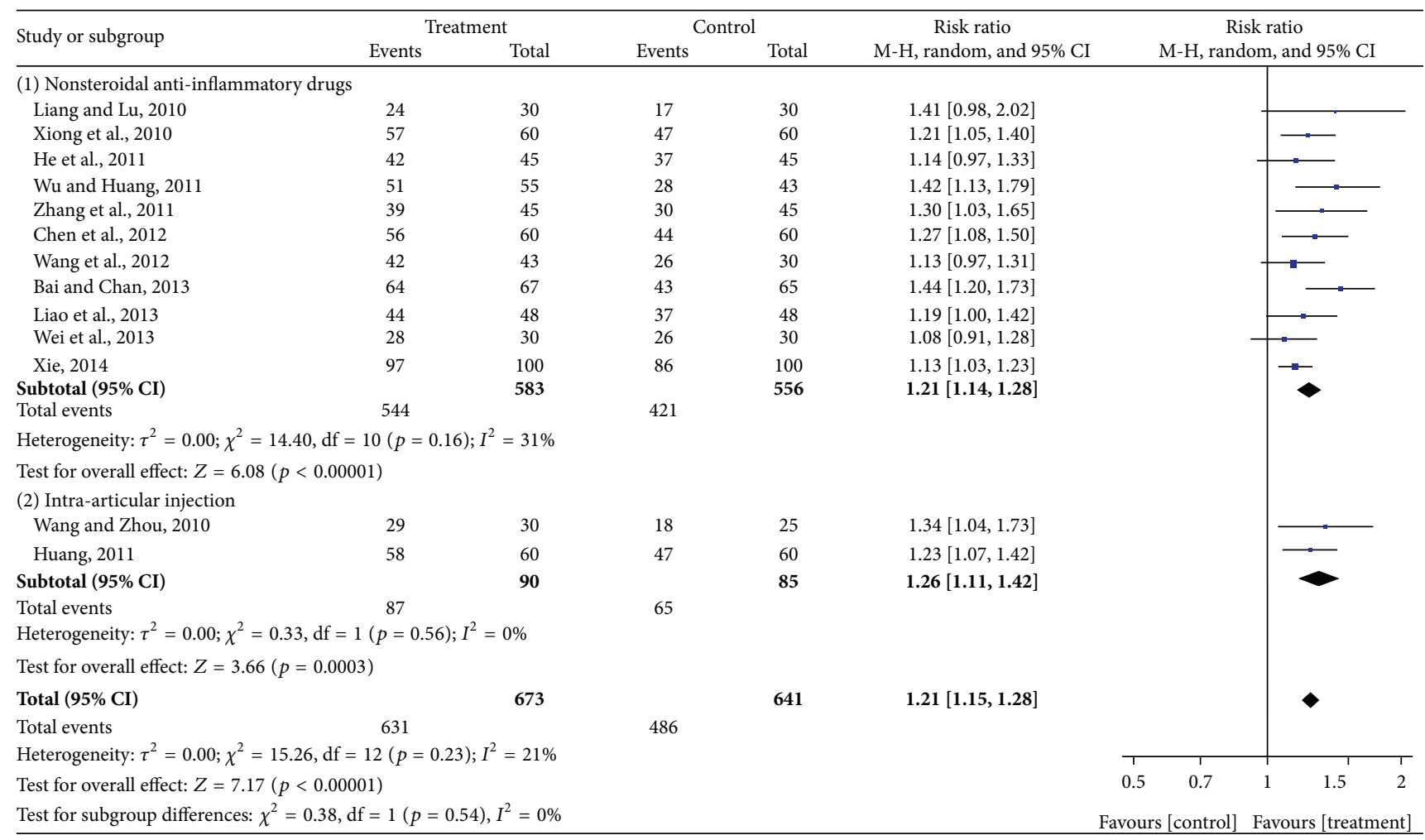

(a) Subgroup analysis by the two different controls

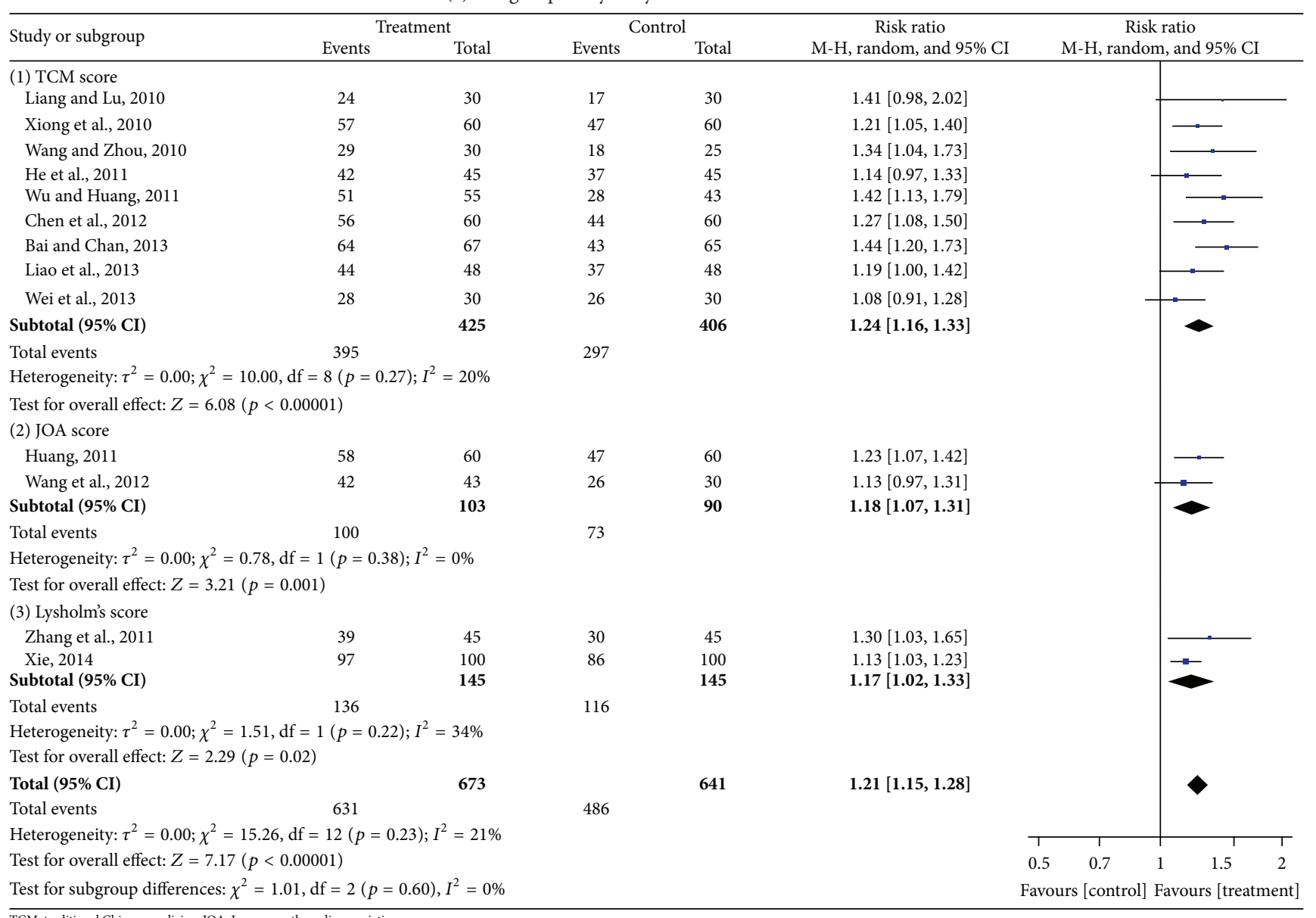

(b) Subgroup analysis by the three different criteria for defining total effectiveness rates

FIGURE 3: Effect of Chinese herbal bath therapy on overall effectiveness. 


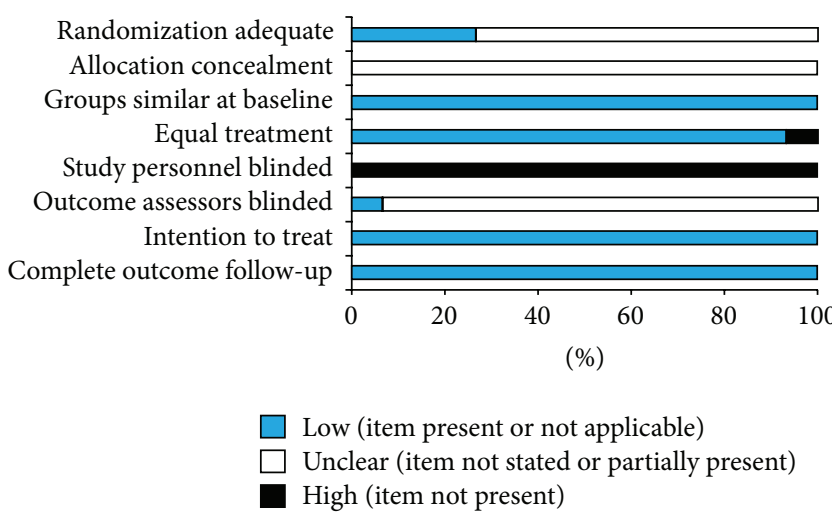

FIGURE 4: Risk of bias for randomized, controlled trials $(n=15)$.

Angilica Root can decrease the levels of interleukin-1 beta (IL-1 $\beta$ ), interleukin (IL)-6, and tumor necrosis factor alpha (TNF- $\alpha$ ) in blood serum [54-56]. Chinese Angelica and Clematis Root similarly can inhibit the IL- $1 \beta$, TNF- $\alpha$, and prostaglandin $\mathrm{E}_{2}\left(\mathrm{PGE}_{2}\right)[57,58]$. Safflower injection has shown optimal therapeutic effect by its reduction of the content of IL-8 and PGE 2 in the knee OA [59]. Argy Wormwood Leaf can protect knee cartilage through regulating the level of the matrix metalloproteinase-13 [60]. These suggest Chinese medicated herbs may have anti-inflammatory effects for patients with arthritis. Cumulatively, these beneficial reports may result in improvements of the clinical symptoms of knee OA.

Adverse events were reported in seven trials, and, of these miscellaneous minor effects, only one patient presented with cutaneous pruritus in the bath treatment groups [25]. Thirtyone patients in the control groups which included loxoprofen, ibuprofen, and meloxicam reported adverse events, such as edema in lower extremities, dizziness, and skin irritation. Thus, during the timeframe of these treatments, Chinese herbal bath therapy appeared to be safer than NSAID interventions.

Our study also has limitations. First, the overall methodological quality of the RCTs was moderate. Many of the trials selected for inclusion contained some methodological deficiencies that might infer high risk of bias. There was no placebo controlled study, no study reported double blinding, and only one admitted single blinding of assessors [16]. We also found that the reporting of procedures in some trials was unclear and insufficient. Second, although meta-analysis showed that the between group difference was statistically significant; the difference between groups is too small to be considered clinically significant. But the total effectiveness rate of herbal bath therapy appeared to demonstrate greater beneficial effects than standard western medication for knee OA. Third, these studies were short-term, whose treatment did not exceed 8 weeks; therefore, longer duration of followups is needed in the future research. Fourth, we did not use statistical methods to test for publication bias due to unanimous publication in Chinese academic journals $[61,62]$ which presents its own difficulties. Many challenges persist, and the potential benefits of Chinese herbal bath therapy for knee OA need to be further evaluated through clinical trials that employ more rigorous methodologies.

\section{Conclusion}

Chinese herbal bath therapy may be effective to reduce the pain and improve the physical functions of knee OA. Despite moderate quality of trials included and the brevity of duration of the intervention, Chinese herbal bath therapy with a history dating back thousands of years radiates a glimmer of hope in the treatment of knee OA. More high quality, rigorously designed and well-controlled RCTs are needed to support the clinical application of Chinese herbal bath therapy for knee OA patients.

\section{Disclaimer}

The contents of this paper are solely the responsibility of the authors and do not necessarily represent the official views of the National Center for Complementary and Integrative Health at National Institutes of Health. The investigators are solely responsible for the content of the paper and the decision to submit for publication. The funders had no role in study design, data collection and analysis, decision to publish, or preparation of the paper.

\section{Conflict of Interests}

The authors declare that there is no conflict of interests regarding the publication of this paper.

\section{Authors' Contribution}

All authors were involved in drafting the paper or revising it critically for important intellectual content, and all authors approved the final version to be published. Chenchen Wang and Bo Chen designed the review protocol. Bo Chen and Xun Lin carried out the literature search. Bo Chen and Min Zhang contributed to data extraction. Bo Chen and Jian Pang contributed to quality assessment. Mei Chung and Hongsheng Zhan provided statistical supports for meta-analysis. Bo Chen performed the analyses and drafted the paper.

\section{Acknowledgments}

Dr. Wang is supported by the National Center for Complementary and Integrative Health at the National Institutes of Health (NCCIH, K24 AT007323). Dr. Chen is supported by the National Natural Science Foundation of China (81202707), by Medical Key Project of Shanghai Science and Technology Committee (12411951400), and by Specialized Research Fund for the Doctoral Program of Higher Education of China (20123107120006). 


\section{References}

[1] L. Fernandes, K. B. Hagen, J. W. J. Bijlsma et al., "EULAR recommendations for the non-pharmacological core management of hip and knee osteoarthritis," Annals of the Rheumatic Diseases, vol. 72, no. 7, pp. 1125-1135, 2013.

[2] D. T. Felson, "Osteoarthritis of the knee," The New England Journal of Medicine, vol. 354, no. 8, pp. 841-848, 2006.

[3] S. S. Lim, T. Vos, A. D. Flaxman et al., "A comparative risk assessment of burden of disease and injury attributable to 67 risk factors and risk factor clusters in 21 regions, 1990-2010: a systematic analysis for the Global Burden of Disease Study 2010," The Lancet, vol. 380, pp. 2224-2260, 2012.

[4] Z. Jotanovic, R. Mihelic, B. Sestan, and Z. Dembic, "Emerging pathways and promising agents with possible disease modifying effect in osteoarthritis treatment," Current Drug Targets, vol. 15, no. 6, pp. 635-661, 2014.

[5] P. M. Barnes, B. Bloom, and R. L. Nahin, "Complementary and alternative medicine use among adults and children: United States, 2007," National Health Statistics Reports, no. 12, pp. 1-23, 2008.

[6] T. Bender, Z. Karagülle, G. P. Bálint, C. Gutenbrunner, P. V. Bálint, and S. Sukenik, "Hydrotherapy, balneotherapy, and spa treatment in pain management," Rheumatology International, vol. 25, no. 3, pp. 220-224, 2005.

[7] M. Karagülle and M. Z. Karagülle, "Effectiveness of balneotherapy and spa therapy for the treatment of chronic low back pain: a review on latest evidence," Clinical Rheumatology, vol. 34, no. 2, pp. 207-214, 2015.

[8] X. L. Zhang, M. F. Zhang, Z. F. Yang et al., "A study on the anti-inflammation and analgesic effects of nature of antirheumatism traditional Chinese medicine," Chinese Archives of Traditional Chinese Medicine, vol. 26, pp. 2386-2396, 2008.

[9] L. Y. Zou, F. Fang, G. Q. Tu et al., "Effect of achyranthes bidentata polysaccharides on hemorrheology of rabbit's knee osteoarthritis," Henan Journal of Traditional Chinese Medicine, vol. 33, pp. 2083-2085, 2013.

[10] T. E. McAlindon, R. R. Bannuru, M. C. Sullivan et al., "OARSI guidelines for the non-surgical management of knee osteoarthritis," Osteoarthritis and Cartilage, vol. 22, no. 3, pp. 363-388, 2014.

[11] L. Y. Liang and M. Q. Lu, "The clinical observation of Wen Jing Tong Luo San for treating knee osteoarthritis induced by wind, cold and dampness," Journal of Chinese Medicinal Materials, vol. 33, pp. 1356-1360, 2010.

[12] Y. Xiong, Z. G. Cheng, C. Y. Peng, and Z. L. Wang, "Miao medicine shengxian soup and herbal fumigation unite treatment knee osteoarthritis 120 cases clinical effects observation," Liaoning Journal of Traditional Chinese Medicine, vol. 37, pp. 2397-2399, 2010.

[13] Y. T. Wang and H. W. Zhou, "The clinical observation of Chinese herbal bath therapy for osteoarthritis of knee joints," Journal of Gansu College of Traditional Chinese Medicine, vol. 27, pp. 4345, 2010.

[14] B. J. He, Z. Fang, G. Y. Xie, and S. Y. Xu, "The effect of steaming and washing with Chinese medicine on the level of MMP-3 and MMP-13 in joint synovia of patients with osteoarthritis," Acta Chinese Medicine and Pharmacology, vol. 39, pp. 61-64, 2011.

[15] C. Q. Huang, “The clinical analysis of Chinese herbs oral and steaming-washing for treating 60 patients with knee osteoarthritis," China Foreign Medical Treatment, vol. 25, pp. 98-99, 2011.

[16] X. Z. Li, “The clinical observation of 'lotion 26 ' for treating degenerative osteoarthritis," Nei Mongol Journal of Traditional Chinese Medicine, vol. 23, pp. 65-66, 2011.

[17] X. P. Wu and X. H. Huang, "The efficacy analysis of acupuncture with Chinese herb fumigating and washing to treat the knee osteoarthritis," Public Medical Forum Magazine, vol. 15, pp. 1154-1155, 2011.

[18] M. X. Zhang, W. J. Xu, and L. X. Li, “The clinical observation of steaming-washing prescription with functional exercise for treating knee osteoarthritis," Shanxi Journal of Traditional Chinese Medicine, vol. 32, article 1649, 2011.

[19] Z. P. Chen, S. Dong, H. Li, and Y. Zhang, "Shujin San fumigating and washing for treating 120 patients with knee osteoarthritis at early and middle stages," Journal of Traditional Chinese Medicine, vol. 53, pp. 1959-1960, 2012.

[20] H. P. Wang, F. L. Liu, and S. Q. Guo, "Chinese herbs fumigating and steaming for treating 43 patients with knee osteoarthritis," Journal of External Therapy of Traditional Chinese Medicine, vol. 21, pp. 20-21, 2012.

[21] K. C. Bai and S. L. Chan, "Santeng Haitongpi decoction local fumigating and washing for treating 67 patients with knee osteoarthritis," Journal of External Therapy of Traditional Chinese Medicine, vol. 22, pp. 14-15, 2013.

[22] H. X. Liao, D. S. Chen, R. L. Yu, C. L. Zhuang, and P. H. Huang, "Clinical research of Chinese herbal bath combined with massage for treating knee osteoarthritis," Journal of Changchun University of Traditional Chinese Medicine, vol. 29, pp. 300-301, 2013.

[23] L. F. Wei, G. P. Xie, H. Lu, and K. X. Zeng, "The clinical observation of Chinese herb fumigating and washing to treat the knee osteoarthritis," Chinese Journal of Ethnomedicine and Ethnopharmacy, vol. 9, pp. 85-87, 2013.

[24] T. Wang, W. J. Hu, Y. S. Peng, Y. Li, and W. Sun, "Gu Bi decoction fumigating and steaming for treating 50 patients with knee osteoarthritis," Chinese Medicine Modern Distance Education, vol. 11, pp. 25-26, 2013.

[25] Z. L. Xie, "Clinical research of Tuina combined with Chinese herbs fumigating and washing to treat the knee osteoarthritis," China Medical Engineering, vol. 22, pp. 72-73, 2014.

[26] Chinese Orthopaedic Association, "Diagnosis and treatment of osteoarthritis," Orthopaedic Surgery, vol. 2, pp. 1-6, 2010.

[27] State Administration of Traditional Chinese Medicine of China, Criteria of Diagnosis and Therapeutic Effect of TCM Diseases, Nanjing University Press, Nanjing, China, 1994.

[28] R. Altman, E. Asch, D. Bloch et al., "Development of criteria for the classification and reporting of osteoarthritis: classification of osteoarthritis of the knee," Arthritis \& Rheumatism, vol. 29, no. 8, pp. 1039-1049, 1986.

[29] M. Okuda, S. Omokawa, K. Okahashi, M. Akahane, and Y. Tanaka, "Validity and reliability of the Japanese Orthopaedic Association score for osteoarthritic knees," Journal of Orthopaedic Science, vol. 17, no. 6, pp. 750-756, 2012.

[30] M. S. Kocher, J. R. Steadman, K. K. Briggs, W. I. Sterett, and R. J. Hawkins, "Reliability, validity, and responsiveness of the Lysholm knee scale for various chondral disorders of the knee," The Journal of Bone \& Joint Surgery-American Volume, vol. 86, no. 6, pp. 1139-1145, 2004. 
[31] R. DerSimonian and N. Laird, "Meta-analysis in clinical trials," Controlled Clinical Trials, vol. 7, no. 3, pp. 177-188, 1986.

[32] J. P. T. Higgins, S. G. Thompson, J. J. Deeks, and D. G. Altman, "Measuring inconsistency in meta-analyses," The British Medical Journal, vol. 327, no. 7414, pp. 557-560, 2003.

[33] G. A. Wells, B. Shea, D. O'Connell et al., “The Newcastle-Ottawa Scale (NOS) for assessing the quality of nonrandomised studies in meta-analyses," Ottawa Hospital Research Institute, 2014, http://www.ohri.ca/programs/clinical_epidemiology/oxford.asp.

[34] G. Guyatt, S. Straus, M. O. Meade et al., "Therapy (randomized trials)," in Users' Guides to the Medical Literature: A Manual for Evidence-Based Clinical Practice, G. Guyatt, D. Rennie, M. O. Meade, and D. J. Cook, Eds., pp. 67-86, McGraw-Hill, New York, NY, USA, 2nd edition, 2008.

[35] Y. K. Ling, Chinese Materia Medica, Shanghai Science and Technology Press, Shanghai, China, 1984.

[36] X. M. Gao, Advanced Series of Books for Chinese Medicine: Chinese Materia Medica, People's Medical Publishing House, Beijing, China, 2000.

[37] M. E. Falagas, E. Zarkadoulia, and P. I. Rafailidis, “The therapeutic effect of balneotherapy: evaluation of the evidence from randomised controlled trials," International Journal of Clinical Practice, vol. 63, no. 7, pp. 1068-1084, 2009.

[38] T. Harzy, N. Ghani, N. Akasbi, W. Bono, and C. Nejjari, "Short- and long-term therapeutic effects of thermal mineral waters in knee osteoarthritis: a systematic review of randomized controlled trials," Clinical Rheumatology, vol. 28, no. 5, pp. 501507, 2009.

[39] A. P. Verhagen, S. Bierma-Zeinstra, J. Lambeck et al., "Balneotherapy for osteoarthritis. A cochrane review," Journal of Rheumatology, vol. 35, no. 6, pp. 1118-1123, 2008.

[40] L. Brosseau, L. MacLeay, V. Robinson et al., "Efficacy of balneotherapy for osteoarthritis of the knee: a systematic review," Physical Therapy Reviews, vol. 7, no. 4, pp. 209-222, 2002.

[41] S. Sukenik, D. Flusser, and M. Abu-Shakra, "The role of Spa therapy in various rheumatic diseases," Rheumatic Disease Clinics of North America, vol. 25, no. 4, pp. 883-897, 1999.

[42] S. Sukenik, M. Abu-Shakra, S. Kudish, and D. Flusser, "Dead Sea and Tiberias as health resort areas for patients suffering from different types of arthritis," Harefuah, vol. 145, no. 2, pp. 117-122, 2006.

[43] A. Fraioli, A. Serio, G. Mennuni et al., "A study on the efficacy of treatment with mud packs and baths with Sillene mineral water (Chianciano Spa Italy) in patients suffering from knee osteoarthritis," Rheumatology International, vol. 31, no. 10, pp. 1333-1340, 2011.

[44] J. Liu, J. Pan, Y. Wang et al., "Component analysis of Chinese medicine and advances in fuming-washing therapy for knee osteoarthritis via unsupervised data mining methods," Journal of Traditional Chinese Medicine, vol. 33, no. 5, pp. 686-691, 2013.

[45] YZ. Bao, SH. Zhang, and WY. Shi, "Percutaneous absorption effects of Chinese herbal medicine," African J Pharm Pharmacol, pp. 389-96, 2012.

[46] R. Rainbow, W. Ren, and L. Zeng, "Inflammation and joint tissue interactions in OA: implications for potential therapeutic approaches," Arthritis, vol. 2012, Article ID 741582, 8 pages, 2012.

[47] C. R. Scanzello and S. R. Goldring, "The role of synovitis in osteoarthritis pathogenesis," Bone, vol. 51, no. 2, pp. 249-257, 2012.
[48] L.-G. Zhang, X.-W. Ouyang, T.-T. Wu, L.-J. Ni, and W.-Z. Shi, "Quantitative evaluation of in vitro effects and interactions of active fractions in a Chinese medicinal formula (Yaotongning Capsule) on rat chondrocytes," Journal of Ethnopharmacology, vol. 155, no. 3, pp. 1424-1432, 2014.

[49] L.-J. Ni, X.-L. Xu, L.-G. Zhang, and W.-Z. Shi, "Quantitative evaluation of the in vitro effect and interactions of active fractions in Yaotongning-based formulae on prostaglandin E2 production," Journal of Ethnopharmacology, vol. 154, pp. 807817, 2014.

[50] J. B. Calixto, M. M. Campos, M. F. Otuki, and A. R. S. Santos, "Anti-inflammatory compounds of plant origin. Part II. Modulation of pro-inflammatory cytokines, chemokines and adhesion molecules," Planta Medica, vol. 70, no. 2, pp. 93-103, 2004.

[51] K. Spelman, J. J. Burns, D. Nichols, N. Winters, S. Ottersberg, and M. Tenborg, "Modulation of cytokine expression by traditional medicines: a review of herbal immunomodulators," Alternative Medicine Review, vol. 11, no. 2, pp. 128-150, 2006.

[52] J.-D. Lee, J.-E. Huh, Y.-H. Baek, K.-C. Cho, D.-Y. Choi, and D.-S. Park, "The efficacy and mechanism action of RvCSd, a new herbal agent, on immune suppression and cartilage protection in a mouse model of rheumatoid arthritis," Journal of Pharmacological Sciences, vol. 109, no. 2, pp. 211-221, 2009.

[53] A. Liacini, J. Sylvester, and M. Zafarullah, "Triptolide suppresses proinflammatory cytokine-induced matrix metalloproteinase and aggrecanase-1 gene expression in chondrocytes," Biochemical and Biophysical Research Communications, vol. 327, no. 1, pp. 320-327, 2005.

[54] P. Ao, Z. G. Zhou, Y. S. Han, L. Y. Yin, and X. Y. Bian, "Effect of chloroform extracts from lycopodium clavatum on RF, TNF$\alpha$, IL-1 $\beta$, IL- 6 of rats with adjuvant arthritis," Information on Traditional Chinese Medicine, vol. 30, pp. 129-131, 2013.

[55] P. Ao, Y. J. Liu, L. Y. Yin, and X. Y. Bian, "Effect of extracts from butyl alcohol of Lycopodium clavatum on serum IL-1 $\beta$, IL-6, TNF- $\alpha$ of rats with adjuvant arthritis," Acta Chinese Medicine and Pharmacology, vol. 39, pp. 22-24, 2011.

[56] Y. J. Liu, L. J. Ma, and X. L. Wang, "Effect of Duhuojisheng decoction on IL-1 and TNF- $\alpha$ from synovial fluid in rabbits with osteoarthritis," Hebei Journal of Traditional Chinese Medicine, vol. 29, pp. 748-750, 2007.

[57] L. X. Yuan, "Effect of Dangguiniantong Tang and decomposition study on IL- $1 \beta$ and TNF- $\alpha$ of rheumatoid arthritis rats," Chinese Archives of Traditional Chinese Medicine, vol. 26, pp. 1213-1214, 2008.

[58] X. S. Zhou, K. Zhou, and F. Feng, "Effect of radix clematidis on IL- $1 \beta$, TNF- $\alpha$ and PGE2 in the rabbit knee osteoarthritis," Lishizhen Medicine and Materia Medica Research, vol. 22, pp. 1143-1144, 2011.

[59] X. D. Jin and M. Zhang, "Experimental study on treatment of osteoarithritis with Honghua injection," Chinese Journal of Traditional Medical Traumatology \& Orthopedics, vol. 17, pp. 1819, 2009.

[60] J. Song, J. R. Yang, F. W. Tian, and N. Li, "Effect of compound artemisiae argvi oil cream on knee cartilage pathological changes and MMP-13 expression in knee osteoarithitis rabbits caused by operation," Journal of Emergency in Traditional Chinese Medicine, vol. 16, pp. 977-979, 2007.

[61] A. Vickers, N. Goyal, R. Harland, and R. Rees, "Do certain countries produce only positive results? A systematic review of 
controlled trials," Controlled Clinical Trials, vol. 19, no. 2, pp. 159-166, 1998.

[62] Y. Y. Wang, L. Q. Wang, Q. Y. Chai, and J. P. Liu, "Positive results in randomized controlled trials on acupuncture published in Chinese journals: a systematic literature review," The Journal of Alternative and Complementary Medicine, vol. 20, article A129, 2014. 


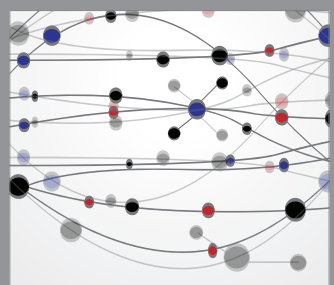

The Scientific World Journal
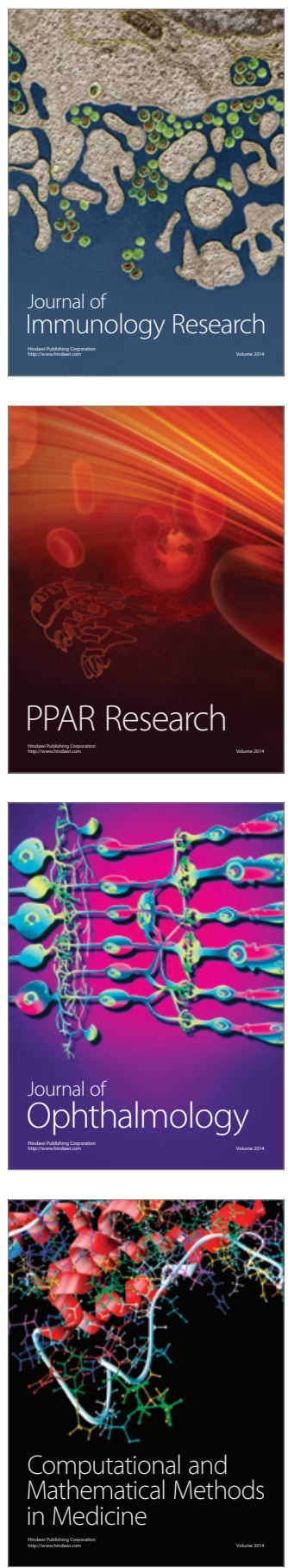

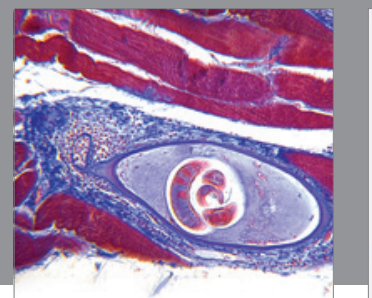

Gastroenterology

Research and Practice
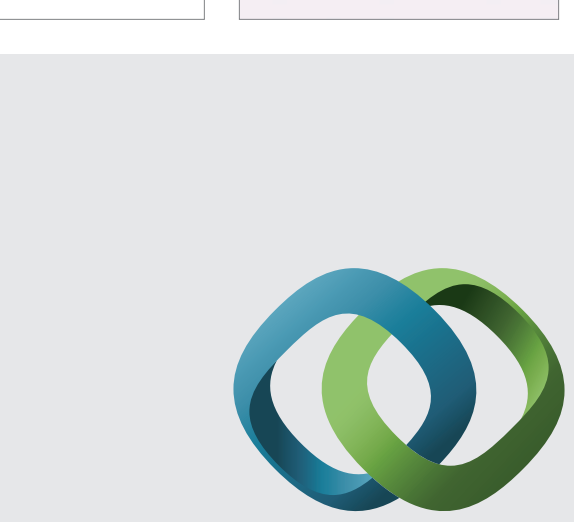

\section{Hindawi}

Submit your manuscripts at

http://www.hindawi.com
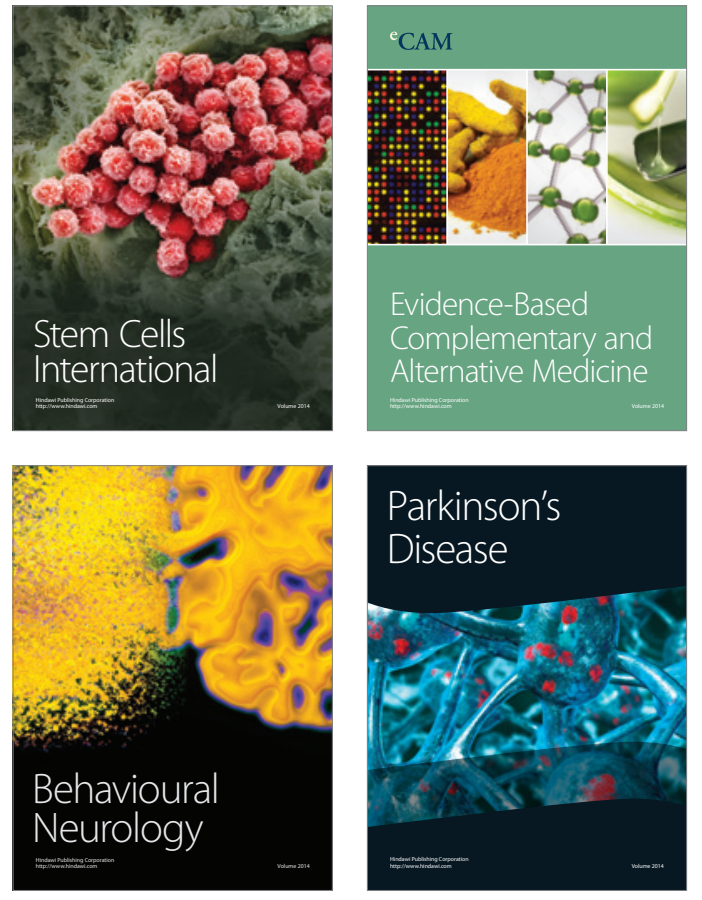
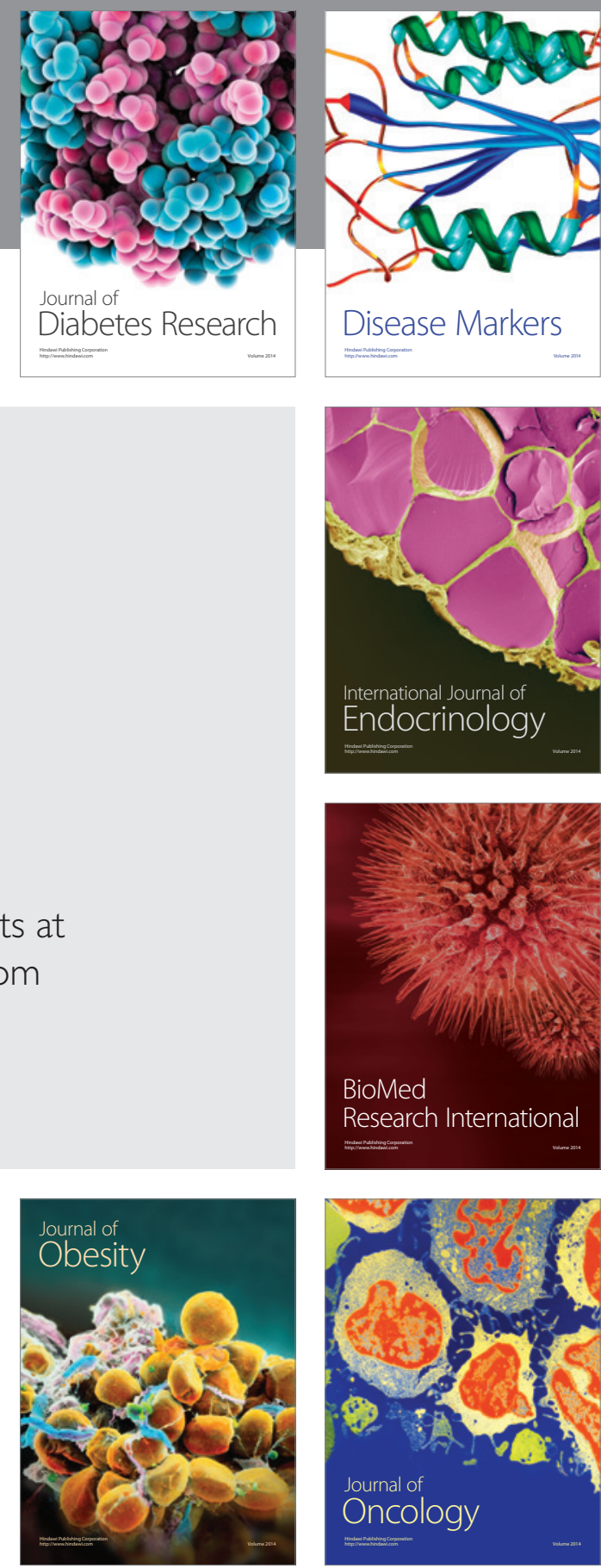

Disease Markers
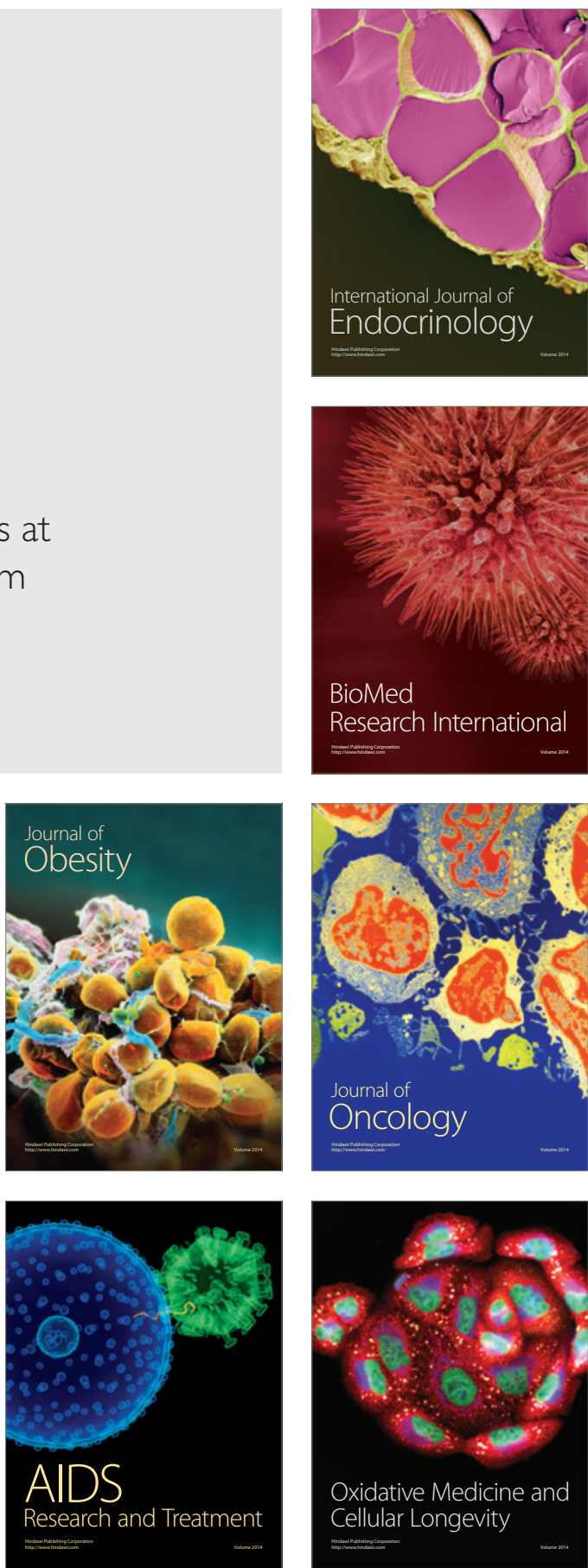